\title{
INNER POINTS AND BREADTH IN CERTAIN COMPACT SEMILATTICES
}

\author{
D. R. BROWN ${ }^{1}$ AND J. W. STEPP
}

\begin{abstract}
A point $x \in X$ is inner if there exists an open set $U$ containing $x$ such that for each open set $V$ with $x \in V \subseteq U$, the inclusion homomorphism $i^{*}$ : $H^{*}(X, X \backslash V) \rightarrow H^{*}(X, X \backslash U)$ is nontrivial. In this note it is proved that, if $X$ is a compact, chainwise connected topological semilattice of codimension $n$, and $x$ is a point of breadth $n+1$, then $x$ is an inner point.
\end{abstract}

1. Introduction. A topological semilattice is a commutative, idempotent topological semigroup; equivalently, it is a partially ordered Hausdorff space in which each pair of elements have a greatest lower bound, and the function $(a, b) \rightarrow \operatorname{glb}\{a, b\}$ is jointly continuous. These objects have been investigated extensively in the past decade, particularly with regard to their relationship to the continuous lattices of D. Scott [5]. In this paper, all semilattices to be discussed are defined on continua, and are additionally assumed to be chainwise connected [8]; that is, if $x<y$, then there exists a subcontinuum $A$ which is simultaneouly a topological arc with endpoints $x$ and $y$ and a totally ordered subset in the order inherited from the space. This subclass, which includes all compact, connected lattices, has received considerable attention over the last several years $[1,3,8,9]$.

In any semilattice $S$, if $x \in S$, then $L(x)=\{y: y \leqslant x\}, M(x)=\{y: y \geqslant x\}$. Since the equivalence mentioned in the first sentence of the paper is established by defining $x \leqslant y$ if $x y=x$, it is immediate that $L(x)=x S$, the principal ideal generated by $x$. No such algebraic alternative exists for $M(x)$; we shall nevertheless refer to this set as the principal filter generated by $x$. By a well-known theorem of $\mathrm{R}$. J. Koch [7] on the existence of arcs in certain partially ordered spaces, it follows that, if $X$ is a continuum and $M(x)$ is connected for each $x \in S$, then $S$ is chainwise connected, as are every $L(x)$ and $M(x)$. Thus $S$ is chainwise connected if and only if $M(x)$ is connected for each $x \in S$, and we shall use these ideas interchangeably.

We shall invoke the cohomology theory of Alexander-Spanier-Wallace, as set forth, for example, in [13]. For compact Hausdorff spaces, this theory satisfies the continuity axiom and is thus equivalent to Čech cohomology. In particular, we will need the exactness of compact pairs, the Mayer-Vietoris Theorem, and the Strong

Received by the editors June 5, 1981 and, in revised form, August 3, 1981; presented to the Society, January 1982, in Cincinatti, Ohio.

1980 Mathematics Subject Classification. Primary 22A26; Secondary 54H12.

Key words and phrases. Inner points, codimension.

${ }^{1}$ Research partially supported by UH Grant REG-1980. 
Excision Theorem which establishes isomorphism between the groups of the (compact) pair $(A \cup B, A)$ and those of $(B, A \cap B)$. The Vietoris Mapping Theorem will be used in the following weak form: if $f: X \rightarrow Y$ is a closed map such that $H^{p}\left(f^{-1}(y)\right)=0$ for all $p \geqslant 0$ (where $H^{0}$ is assumed reduced), then $f^{*}$ is an isomorphism in all dimensions. For a proof of this theorem in ASW cohomology and in much greater generality, see [10]. Our codimension function is that of Haskell Cohen [4]: $\operatorname{cd}(X) \leqslant n$ provided $i^{*}: H^{n}(X) \rightarrow H^{n}(A)$ is onto for every closed subset $A$ of $X$. A nonempty space is acyclic provided it has trivial reduced cohomology; that is, all of its groups are trivial. Thus, an acyclic space has codimension $\leqslant n$ if its closed subsets all have trivial $n$-cohomology. Finally, we shall have occasion to use the following corollary of Wallace's Acyclicity Theorem [15]: a compact, connected topological semilattice is acyclic.

The breadth of a semilattice $S$, denoted $\operatorname{Br}(S)$, is the smallest positive integer $n$ such that any finite subset $F$ of $S$ has a subset $F_{1}$ of at most $n$ elements such that $\inf \left(F_{1}\right)=\inf (F)$. Semilattices of finite breadth have received periodic attention in the literature $[2,8,9]$. Lawson $[8$, p. $210 ; 9$, p. 557] proved these inequalities for a large class of topological semilattices which includes the compact, chainwise connected ones: $\operatorname{cd}(S) \leqslant \operatorname{Br}(S) \leqslant \operatorname{cd}(S)+1$, where the first relation becomes equality if $S$ has an identity. Thus a compact, connected lattice $L$ has $\operatorname{cd}(L)=\operatorname{Br}(L)$, but there exist chainwise connected semilattices of codimension $n$ and breadth $n+1$. Indeed, if $I=[0,1]$ with the usual order, then $S=\{(x, y) \in I \times I: x y=0\}$ is an example for $n=1$. A more motivating example, where $n=2$, is given in [3, p. 41].

The idea of an intrinsic boundary for topological spaces has existed for quite a long time, particularly in topological algebra. An informal survey of the history of this subject up until 1965 occurs in [6]; subsequently, Lawson and Madison [11, 12] weakened previous definitions to the following. A point $x \in X$ is peripheral if, for each open set $U$ containing $x$, there exists an open set $V$ containing $x, V \subseteq U$, such that the inclusion homomorphism $i^{*}: H^{p}(X, X \backslash V) \rightarrow H^{p}(X, X \backslash U)$ is trivial in all dimensions. Points which are not peripheral are called inner points. This note proves the following.

THEOREM. Let $S$ be a compact, chainwise connected topological semilattice of codimension $n$. If $\operatorname{Br}(S)=n+1$, then any point of breadth $n+1$ is an inner point.

Points of breadth $n+1$ are, of course, those points possessing an irreducible representation as a product of $n+1$ elements. The uniqueness of this result lies in the following observation: previously, theorems involving algebraic properties of elements and their consequent positions have concluded such elements to be peripheral. For example, maximal elements in compact semilattices are peripheral and the identity of a clan is peripheral [12]. To the authors' knowledge, the theorem above is the first one in which algebraic conditions on an element force it to be an inner point. 


\section{Technical considerations.}

LEMma 1. Let $X$ be an acyclic space. Then $x \in X$ is an inner point of $X$ if and only if there exists an open set $U$ containing $x$ such that for each open set $V$ containing $x$, $V \subseteq U$, the homomorphism induced by the inclusion map $j: X \backslash U \rightarrow X \backslash V$ is nonzero in some dimension.

Proof. By the acyclicity of $X$ and the exactness of the pair sequences $(X, X \backslash U)$ and $(X, X \backslash V)$, the horizontal arrows in the diagram below are isomorphisms in all dimensions:

$$
\begin{array}{ccc}
H^{p}(X \backslash V) & \rightarrow & H^{p+1}(X, X \backslash V) \\
j^{*} \downarrow & & i^{*} \downarrow \\
H^{p}(X \backslash V) & \rightarrow & H^{p+1}(X, X \backslash U)
\end{array}
$$

The conclusion follows from independence of path and the observation that $x$ is an inner point if and only if $i^{*}$ is nonzero in some dimension.

LEMMA 2. Let $A$ be a closed subset of $X$, a compact Hausdorff space. Let $\operatorname{cd}(X)=n$, $H^{n}(X)=0=H^{n-1}(A)$. If $x$ is an inner point in dimension $n-1$ relative to $A$, then $X$ is an inner point relative to $X$ (in the same dimension).

Proof. By the previous lemma, there exists a set $U$, open in $A$ and containing $x$, such that $j^{*}: H^{n-1}(A \backslash V) \rightarrow H^{n-1}(A \backslash U)$ is not trivial for every $A$-open set $V \subseteq U$ such that $x \in V$.

Let $U_{1}$ be an open set in $X$ such that $U_{1} \cap A=U$, and let $V_{1}$ be any open set in $X$ such that $x \in V_{1} \subseteq U_{1}$. Consider the Mayer-Vietoris exact sequence:

$$
H^{n-1}\left(X \backslash V_{1}\right) \times H^{n-1}(A) \rightarrow H^{n-1}\left(\left(X \backslash V_{1}\right) \cap A\right) \rightarrow H^{n}\left(A \cup\left(X \backslash V_{1}\right)\right) .
$$

Since $\operatorname{cd}(X)=n$ and $H^{n}(X)=0$, the final group in the above triple is zero. Because $H^{n-1}(A)=0$, it follows that $i_{1}^{*}$, the homomorphism induced by the inclusion of $A \backslash V_{1}$ into $X \backslash V_{1}$, is onto $H^{n-1}\left(A \backslash V_{1}\right)$. Since $A \cap V_{1}$ is an $A$-open set contained in $U$ and containing $x, j^{*}$ is not zero. Finally, from the diagram

$$
\begin{array}{ccc}
H^{n-1}\left(X \backslash V_{1}\right) & \stackrel{j_{1}^{*}}{\rightarrow} & H^{n-1}\left(X \backslash U_{1}\right) \\
i_{1}^{*} \downarrow & & i_{U}^{*} \downarrow \\
H^{n-1}\left(A \backslash V_{1}\right) & \stackrel{j^{*}}{\rightarrow} & H^{n-1}(A \backslash U),
\end{array}
$$

we have $0 \neq j^{*} i_{1}^{*}=i_{U}^{*} j_{1}^{*}$, whence $j_{1}^{*} \neq 0$, which shows that $x$ is an inner point relative to $X$.

Lemma 3. Let $\left\{A_{i}\right\}_{i=1}^{n}$ be a collection of acyclic sets such that $\bigcap_{i \in I} A_{i}$ is acyclic for every nonempty $I \subseteq\{1, \ldots, n\}$. Then $\cup\left\{A_{i}: i=1, \ldots, n\right\}$ is acyclic.

Proof. Each $A_{i}$ is connected, as are pairwise intersections; this proves the lemma in dimension 0 , for all $n$. If $n=2$, the result is immediate by Mayer-Vietoris in all dimensions. If $n>2$, the lemma follows by direct application of mathematical induction. 
LEMMA 4. Let $S$ be a compact, chainwise connected semilattice with $\operatorname{cd}(S)=n$. Let $z$ be an element of breadth $n+1$, with irreducible representation $z=x_{1} x_{2} \cdots x_{n+1}$. Let $y_{i}=x_{1} x_{2} \cdots \hat{x}_{i} \cdots x_{n+1}$, where " $\hat{x}$ " means "delete $x$ ". Then, for any proper subset $I \subseteq\{1, \ldots, n+1\}, \cap\left\{M\left(y_{i}\right): i \in I\right\} \neq \square$, whereas

$$
\bigcap\left\{M\left(y_{i}\right): i=1, \ldots, n+1\right\}=\square .
$$

Proof. Choose $j \in\{1, \ldots, n+1\} \backslash I$. Then $x_{j} \geqslant y_{i}$ for every $i \in I$. Hence $\cap\left\{M\left(y_{i}\right): i \in I\right\} \neq \square$.

Next, suppose there exists $a \in \bigcap\left\{M\left(y_{i}\right): i=1, \ldots, n+1\right\}$. Let $b_{i}=a x_{i}, i=$ $1, \ldots, n+1$. If $b_{i}=b_{j}, i \neq j$, then $a x_{i} x_{j}=a x_{j}$, whence $z=a z=a x_{1} \cdots x_{n+1}=a y_{i}$ $=y_{i}$, which contradicts the irreducible representation of $z=x_{1} \cdots x_{n+1}$. Hence all $b_{i}$ 's are distinct. Now note $b_{1} b_{2} \cdots b_{n+1}=a\left(x_{1} \cdots x_{n+1}\right)=a z=z$. The chainwise connected semilattice $a S$ has identity $a$ and is of codimension less than or equal to $n$; as mentioned before, $\operatorname{Br}(a S)=n$, so that $z$ may be written as the product of a proper subset of $\left\{b_{1}, \ldots, b_{n+1}\right\}$. Without loss of generality, assume $z=b_{1} \cdots b_{n}$. Then $z=a\left(x_{1} \cdots x_{n}\right)=a y_{n+1}=y_{n+1}$. This again contradicts the irreducible representation of $z$ as the product $x_{1} \cdots x_{n+1}$.

The next lemma is vintage algebraic topology, in the spirit of finite simplicial complexes, and a proof can be constructed from the definition of cohomology groups. For the sake of brevity, we give a proof using Lemma 3.

LEMMA 5. Let $\left\{A_{1}, \ldots, A_{n+1}\right\}$ be a collection of distinct, compact, acyclic sets such that $\cap\left\{A_{i}: i \in I\right\}$ is acyclic and nonempty for each $I$ properly contained in $\{1, \ldots, n$ $+1\}$, but $\cap\left\{A_{i}: i=1,2, \ldots, n+1\right\}=\square$. Then

$$
H^{n-1}\left(A_{1} \cup \cdots \cup A_{n+1}\right) \neq 0 \text {. }
$$

Proof. Note that $I \neq J \subseteq\{1, \ldots, n+1\}$ implies

$$
\bigcap\left\{A_{i}: i \in I\right\} \neq \bigcap\left\{A_{j}: j \in J\right\} \text {. }
$$

If $n=1$, then $\tilde{H}^{0}\left(A_{1} \cup A_{2}\right) \neq 0$, since $A_{1} \cap A_{2}=\square$. Suppose the conclusion is valid for all families of $k$ sets satisfying the hypotheses, and let $\left\{A_{i}\right\}_{i=1}^{k+1}$ be a family of $k+1$ sets which satisfy the hypotheses. Consider the Mayer-Vietoris sequence:

$$
\begin{gathered}
H^{k-2}\left(\bigcup_{i=1}^{k} A_{i}\right) \times H^{k-2}\left(A_{k+1}\right) \rightarrow H^{k-2}\left(\bigcup_{i=1}^{k}\left(A_{i} \cap A_{k+1}\right)\right) \\
\Delta \swarrow \\
H^{k-1}\left(\bigcup_{i=1}^{k+1} A_{i}\right) \rightarrow H^{k-1}\left(\bigcup_{i=1}^{k} A_{i}\right) \times H^{k-1}\left(A_{k+1}\right) .
\end{gathered}
$$

By Lemma 3, $\cup\left\{A_{i}: i=1, \ldots, k\right\}$ is acyclic, so that $\Delta$ is an isomorphism. For $i=1, \ldots, k$, set $B_{i}=A_{i} \cap A_{k+1}$ and note that if $i \neq j$, then $B_{i} \neq B_{j}$. Let $I \subseteq$ $\{1, \ldots, k\}$; then $\cap\left\{B_{i}: i \in I\right\}=\bigcap\left\{A_{j}: j \in I \cup\{k+1\}\right\}$. Hence $\cap\left\{B_{i}: i=\right.$ $1, \ldots, k\}=\square$ and $\cap\left\{B_{i}: i \in I\right\}$ is acyclic (and nonempty) for every $I$ properly 
contained in $\{1, \ldots, k\}$. By the inductive hypothesis, $H^{k-2}\left(\cup_{i=1}^{k} B_{i}\right) \neq 0$, so that $H^{k-1}\left(\cup_{i=1}^{k+1} A_{i}\right) \neq 0$.

3. Proof of the theorem. Let $X$ be a compact, chainwise connected topological semilattice, $\operatorname{cd}(X)=n$. Let $z$ be an element of breadth $n+1$ with irreducible representation $z=x_{1} x_{2} \cdots x_{n+1}$. We first reduce $X$ to more manageable size. Fix $i \in\{1, \ldots, n+1\}$; for $j \neq i$, let $C_{i j}$ be an arc-chain with endpoints $x_{i}$ and $x_{i} x_{j}$. Let $A_{i}=\Pi\left\{C_{i j} \mid j \neq i\right\}$. Note that each $A_{i}$ contains all finite products of the $x_{j}$ 's which contain $x_{i}$ as a factor. Finally, let $S$ be the semilattice generated by $\cup\left\{A_{i}: i=1, \ldots, n+1\right\}$. It is easily verified that $S$ is compact, chainwise connected, with maximal elements $x_{1}, \ldots, x_{n+1}$ and minimal element $z$. Since $\operatorname{Br}(S)=n+1$, $\operatorname{cd}(S)=n$. We will show that $z$ is an inner point relative to $S$, and appeal to Lemma 2 to complete the argument. To this end, all further calculations are performed within $S$.

As in the proof of Lemma 4, let $y_{i}=x_{1} x_{2} \cdots \hat{x}_{i} \cdots x_{n+1}$. Let

$$
B=\bigcup\left\{M\left(y_{i}\right): i=1, \ldots, n+1\right\} .
$$

By Lemmas 4 and $5, H^{n-1}(B) \neq 0$. Let $U=S \backslash B$; we show this set satisfies the hypothesis of Lemma 1 . For any open set $W$ containing $z, W \subseteq U$, there exists an open set $V$ containing $z, V \subseteq W$, such that $S \backslash V=\cup\left\{M\left(q_{j}\right): j=1, \ldots, h\right\}$ [14]. As $S \backslash U=B \subseteq S \backslash W \subseteq S \backslash V$, it clearly suffices to show $j^{*}: H^{n-1}(S \backslash V) \rightarrow$ $H^{n-1}(S \backslash U)$ is not trivial.

For ease of notation, let $a=x_{1}$ henceforth. Let $\lambda_{a}: S \rightarrow a S$ by $\lambda_{a}(t)=a t$, let $\lambda_{1}=\lambda_{a} \mid S \backslash V$ and $\lambda=\lambda_{a} \mid B$. The following diagram is independent of path:

$$
\begin{array}{ccc}
H^{n-1}(a(S \backslash V)) & \stackrel{\lambda_{1}^{*}}{\rightarrow} & H^{n-1}(S \backslash V) \\
j_{1}^{*} \downarrow & & j^{*} \downarrow \\
H^{n-1}(a B) & \stackrel{\lambda^{*}}{\rightarrow} & H^{n-1}(B)
\end{array}
$$

In order to have $j^{*} \neq 0$, it suffices to show that $\lambda^{*}$ is an isomorphism and $j_{1}^{*} \neq 0$.

The former result is accomplished via the Vietoris Mapping Theorem. Fix $x \in a B$; we show $\lambda^{-1}(x)$ is an acyclic subset of $B$. Clearly $\lambda^{-1}(x) \cap M\left(y_{i}\right)=\lambda_{a}^{-1}(x) \cap M\left(y_{i}\right)$. Let $\Lambda \subseteq\{2, \ldots, n+1\}$ be defined by $i \in \Lambda$ if and only if $\lambda^{-1}(x) \cap M\left(y_{i}\right) \neq \square$. Thus $\lambda^{-1}(x)=\left[\lambda^{-1}(x) \cap M\left(y_{1}\right)\right] \cup\left[\cup_{i \in \Lambda}\left(\lambda^{-1}(x) \cap M\left(y_{i}\right)\right)\right]$. Note that the second of the two bracketed sets is, in fact, $\lambda^{-1}(x) \cap\left(\cap_{i \in \Lambda} M\left(y_{i}\right)\right)$. To see this, fix $t \in \cup_{i \in \Lambda}\left(\lambda^{-1}(x) \cap M\left(y_{i}\right)\right)$; it suffices to show $t \geqslant y_{i}$ for each $i \in \Lambda$. Fix $i \in \Lambda$, let $s \in \lambda^{-1}(x) \cap M\left(y_{i}\right)$. Then, since $i \neq 1, a \in M\left(y_{i}\right)$ also, whence $x=a s \in M\left(y_{i}\right)$. Thus $y_{i} \leqslant x=a t \leqslant t$. Since $\lambda^{-1}(x) \cap M\left(y_{i}\right)=\lambda_{a}^{-1}(x) \cap M\left(y_{i}\right)$ and $\lambda_{a}^{-1}(x)$ is a compact, chainwise connected semilattice, $\lambda^{-1}(x) \cap M\left(y_{i}\right)$ is a principal filter in $\lambda_{a}^{-1}(x)$ and is therefore acyclic. In a similar manner, $\lambda^{-1}(x) \cap\left(\cap_{i \in \Lambda} M\left(y_{i}\right)\right)$, iff nonempty, is acyclic.

Finally, if both $\lambda^{-1}(x) \cap M\left(y_{1}\right)$ and $\lambda^{-1}(x) \cap\left(\cap_{i \in \Lambda} M\left(y_{i}\right)\right)$ are nonempty, then choose $t$ in the first set, $s$ in the second. Since $x=a t=a s$, and $a \in \cap_{i \in \Lambda} M\left(y_{i}\right)$, 
we have $x \in \cap_{i \in \Lambda} M\left(y_{i}\right)$, from which it follows that $t \in \cap_{i \in \Lambda} M\left(y_{i}\right) \cap \lambda^{-1}(x)$. Hence, in this case the first set is a subset of the second, so that the intersection is again acyclic. By Lemma 3, $\lambda^{-1}(x)$ is acyclic; by the Vietoris Mapping Theorem, $\lambda^{*}$ is therefore an isomorphism in all dimensions.

We will invoke a similar technique to establish that $j_{1}^{*} \neq 0$; however, the use of Lemma 3 is somewhat more delicate than above. Since $\lambda^{*}$ is an isomorphism and $H^{n-1}(B) \neq 0$, it suffices to show that $j_{1}^{*}$ is surjective. By exactness of the pair sequence $(a(S \backslash V), a B)$, this can be accomplished by showing $H^{n}(a(S \backslash V), a B)=0$. From the Strong Excision Theorem [13], decomposing $a(S \backslash V)$ as $[(S \backslash V) \cap L(a)$ ] $\cup a B, H^{n}(a(S \backslash V), a B) \cong H^{n}((S \backslash V) \cap L(a),(S \backslash V) \cap a B)$. We show the latter group to be trivial by showing that each factor is acyclic and again appealing to the exactness of pair sequences.

(i) $a(S \backslash V)=((S \backslash V) \cap L(a)) \cup a B$. This is a consequence of the construction of $S$. Let $x=a t$, with $t \in S \backslash V$. If $t \in L(a)$, then $x=a t=t$; hence it must be shown that, if $t \notin L(a)$, then $a t \in a B$. Let $t=t_{1} \cdots t_{m}$, where each $t_{k}$ belongs to some $C_{i j}$, an arc-chain with endpoints $x_{i}$ and $x_{i} x_{j}$. Since $t \notin L(a), i \neq 1$ for each upper endpoint $x_{i}$. If, for some $j, x_{j}=1$, then $a\left(x_{i} x_{j}\right) \leqslant a t_{k} \leqslant a x_{i}$, whence $a t_{k}=a x_{i}$ $\in a M\left(y_{1}\right)$. If $x_{j} \neq a$, then $y_{1} \leqslant x_{i} x_{j}$, so that again $a t_{k} \in a M\left(y_{1}\right)$. Thus $a t=a t_{1}$ $\cdots t_{m}=\left(a t_{1}\right) \cdots\left(a t_{m}\right) \in a M\left(y_{1}\right) \subseteq a B$. Right to left inclusion is immediate.

(ii) $(S \backslash V) \cap L(a)$ is acyclic. By construction, $S \backslash V$ is the finite union of principal filters; cut down to $L(a)$, a chainwise connected semilattice, they retain this property. The intersection of all of these filters (which meet $L(a)$ at all) must contain $a$ and is therefore itself a principal filter in $L(a)$. By Lemma 3 , the set $(S \backslash V) \cap L(a)$ is acyclic.

(iii) $(S \backslash V) \cap a B$ is acyclic. If $n=1$, then $B=\left\{x_{1}=a, x_{2}\right\}$, so that $(S \backslash V) \cap a B$ $=\{a\}$ in this case. We assume henceforth that $n \geqslant 2$. For $2 \leqslant j \leqslant n+1$, define

$$
\begin{gathered}
T_{j}=(S \backslash V) \cap a\left[M\left(y_{1}\right) \cap L\left(x_{j}\right)\right], \\
S_{j}=\bigcup_{k \neq 1, j}\left[M\left(y_{k}\right) \cap L(a)\right], \quad J_{j}=S_{j} \cup T_{j} .
\end{gathered}
$$

A direct argument establishes $(S \backslash V) \cap a B=\cup\left\{J_{j}: j \geqslant 2\right\}$. We show that the family of $J_{j}$ 's satisfies the hypotheses of Lemma 3. Each $S_{j}$ is the union of principal filters in $L(a)$, hence is acyclic by Lemma 3 . In a similar manner, each $T_{j}$ is the union of finitely many principal filters in the chainwise connected subsemilattice $a\left[M\left(y_{1}\right) \cap L\left(x_{j}\right)\right]$, even though some of the $M\left(q_{i}\right)$ 's which comprise $S \backslash V$ will fail to intersect this set. Thus $T_{j}$ is also acyclic by Lemma 3 . The set $S_{j} \cap T_{j}$ is, like $T_{j}$, the finite union of principal filters in $a\left[M\left(y_{1}\right) \cap L\left(x_{j}\right)\right]$, and hence acyclic. Once more applying Lemma 3 , we have $J_{j}$ acyclic. Next fix $\Lambda$, a nonempty subset of $\{2, \ldots, n+1\}$, and let $J_{\Lambda}=\bigcap\left\{J_{j}: j \in \Lambda\right\}, T_{\Lambda}=\bigcap\left\{T_{j}: j \in \Lambda\right\}$, and $S_{\Lambda}=$ $\cap\left\{S_{j}: j \in \Lambda\right\}$. Finally, for $\Lambda^{\prime} \subseteq \Lambda$, let $F_{\Lambda^{\prime}}=S_{\Lambda^{\prime}} \cap_{T \Lambda \backslash \Lambda^{\prime}}$. It is a tautology that $J_{\Lambda}=\bigcup\left\{F_{\Lambda^{\prime}}: \Lambda^{\prime} \subseteq \Lambda\right\}$, and that $F_{\Lambda} \cap F_{\Omega}=F_{\Lambda \cup \Omega}$ for any subsets $\Lambda, \Omega \subseteq\{2, \ldots, n$ +1 \}. To prove $J_{\Lambda}$ is acyclic, it suffices to prove each $F_{\Lambda^{\prime}}$ is acyclic, that $F_{\Lambda}$ is nonempty, then invoke Lemma 3 again. Now $S_{\Lambda^{\prime}}$ remains the finite union of 
principal filters in $L(a)$, whereas $T_{\Lambda \backslash \Lambda^{\prime}}=(S \backslash V) \cap a\left[M\left(y_{1}\right) \cap L(w)\right]$, where $w=$ $\Pi\left\{x_{j}: j \in \Lambda \backslash \Lambda^{\prime}\right\}$. Because $w a \leqslant a$, we may therefore regard $F_{\Lambda^{\prime}}=S_{\Lambda^{\prime}} \cap T_{\Lambda \backslash \Lambda^{\prime}}$ as the union of principal filters in the chainwise connected semilattice $a\left[M\left(y_{1}\right) \cap L(w)\right]$, and thus $F_{\Lambda^{\prime}}$ is acyclic by Lemma 3. Since $a \in S_{\Lambda}=F_{\Lambda}$, the set $F_{\Lambda}$ is nonempty. This establishes that the family $\left\{F_{\Lambda^{\prime}}: \Lambda^{\prime} \subseteq \Lambda\right\}$ satisfies the hypotheses of Lemma 3, so that $J_{\Lambda}$ is itself acyclic. Hence the family $\left\{J_{j}: j \geqslant 2\right\}$ also satisfies the conditions of Lemma 3, from which we conclude at last that $(S \backslash V) \cap a B$ is acyclic.

From our earlier remarks, $j_{1}^{*} \neq 0$, whence $j^{*} \neq 0$, and the proof of the theorem is complete.

In closing, it is a pleasure to record our indebtedness to our colleague, Michael Friedberg, for his patient listening and valuable comments.

\section{REFERENCES}

1. L. W. Anderson, On the breadth and co-dimension of a topological lattice, Pacific J. Math. 9 (1959), 327-333.

2. K. Baker and A. R. Stralka, Compact, distributive lattices of finite breadth, Pacific J. Math. 34 (1970), $311-320$.

3. D. R. Brown, Topological semilattices on the two-cell, Pacific J. Math. 15 (1965), 36-46.

4. H. Cohen, A cohomological definition of dimension for locally compact Hausdorff spaces, Duke Math. J. 21 (1954), 209-224.

5. G. Gierz, K. H. Hofmann, K. Keimel, J. D. Lawson, M. Mislove and D. Scott, A compendium of continuous lattices, Heidelberg, 1980.

6. K. H. Hofmann and P. S. Mostert, Elements of compact semigroups, Merrill, Columbus, Ohio, 1966.

7. R. J. Koch, Arcs in partially ordered spaces, Pacific J. Math. 9 (1959), 723-728.

8. J. D. Lawson, The relation of breadth and codimension in topological semilattices, Duke Math. J. 37 (1970), 207-212.

9. The relation of breadth and codimension in topological semilattices. II, Duke Math. J. 38 (1971), 555-559.

10. _ A generalized version of the Vietoris-Begle theorem, Fund. Math. 55 (1969), 65-72.

11. J. D. Lawson and B. Madison, Peripheral and inner points, Fund. Math. 69 (1970), 253-266.

12. __ Peripherality in semigroups, Semigroup Forum 1 (1970), 128-142.

13. E. H. Spanier, Algebraic topology, McGraw-Hill, New York, 1966.

14. J. W. Stepp, Semilattices which are embeddable in a product of min intervals, Proc. Amer. Math. Soc. 28 (1971), 81-86.

15. A. D. Wallace, Acyclicity of compact connected semigroups, Fund. Math. 50 (1961), 99-105.

DeParTment of MATHEMATICS, University OF Houston, Houston, TeXas 77004 